\title{
Enfoque cognitivo para la comprensión de narraciones: una mirada desde la psicología discursiva y el modelo de indexación de eventos*
}

\author{
Violeta Cautín-Epifani**
}

\begin{abstract}
Resumen
El presente artículo tiene como objetivo realizar una revisión de la postura cognitiva respecto de la narración desde donde se propone que existirían elementos que permiten afirmar que este modo de organización textual responde a procesos naturales internos que le favorecen. Nos centraremos principalmente en realizar un recorrido por dicha postura para llegar a analizar en profundidad uno de los modelos de comprensión de la narración planteados desde la psicología discursiva, el modelo de indexación de eventos (Zwaan, Langston \& Graesser, 1995a). Concluimos nuestra revisión señalando los aportes y desafíos que presenta esta visión tanto para futuras investigaciones en el área, como para la didáctica de la comprensión textual.
\end{abstract}

Palabras clave: comprensión, narración, modelo de situación, modelo de indexación de eventos.

\section{Discursive psychology and the event- indexing model}

\begin{abstract}
The following article perfoms a review of the cognive vision regarding narration. This vision claims that narration responds to internal natural processes that favors aids its development and comprehension. We analyse this vision from its begining until we focus and examine one of the comprehension models of narration that emerge from discursive psychology: the event indexing model (Zwaan, Langston \& Graesser, 1995a). We finish our bibliographic investigation pointing at the benefits and challenges that this model presents not only for future research in the area, but also for the didactics of textual comprehension.
\end{abstract}

Key words: comprehension, narration, situation model, event indexing model.

Recibido: 20-08-2013

Aceptado: 29-10-2013

* Artículo posible gracias al apoyo de CONICYT.

** Chilena, Doctora (c) en Lingüística, Pontificia Universidad Católica de Valparaíso, Valparaíso, Chile, violeta.cautin@gmail.com 


\section{Introducción}

Desde el enfoque cognitivo y en especial del procesamiento del discurso, la comprensión de la narración ha sido ampliamente estudiada, la forma en que las personas comprenden narraciones o historias simples (Johson-Laird, 1983; McKoon \& Ratcliff, 1992; Sanford \& Garrod, 1981; Van Dijk \& Kintsch, 1983; Yarkoni, Speer \& Zacks, 2008; Zwaan, Langston \& Graesser, 1995; Zwan, Magliano \& Graesser, 1995; Zwaan \& Radvansky, 1998).

Entre los investigadores de esta línea existe consenso en afirmar que a medida que el lector va comprendiendo la narración, construye una representación mental de la situación descrita en la misma, lo que se conoce como 'modelo de situación' (Van Dijk \& Kintsch, 1983; Graesser, Singer \& Trabasso, 1994; Zwaan \& Radvansky, 1998). Dicha representación juega un papel fundamental no solo en la comprensión de la narración, sino también en su recuerdo, ya que, según lo planteado por estos investigadores, es desde este 'modelo de situación' que las personas extraemos nuestros recuerdos de lo leído (Yarkoni et al., 2008). Sin embargo, pese a que se reconoce la construcción e importancia fundamental de dicho nivel representacional durante la comprensión de narraciones, su naturaleza exacta y en consecuencia, la de la información contenida en él, es todavía materia de discusión (Graesser \& WiemerHasting, 1999; Therriault \& Rinck, 2007).

Una de las propuestas que ha recibido bastante apoyo por parte de la psicología discursiva se refiere a la naturaleza 'multidimensional' de dicha representación, es decir, estas serían construidas y actualizadas teniendo en cuenta diferentes dimensiones o índices (indexes) los cuales el lector monitorea durante la lectura de la narración (Zwaan et al., 1995a; Zwaan et al., 1995b; Zwaan \& Radvansky, 1998). Dichas dimensiones jugarían un papel crucial en el proceso de comprensión y recuerdo de las narraciones, ya que, dadas ciertas condiciones, los investigadores postulan que facilitarían o dificultarían dichos procesos.

En este sentido, el presente artículo analizará, a partir de una revisión bibliográfica, el enfoque multidimensional en la construcción de dicho modelo de situación, centrándonos en la propuesta del modelo de indexación de eventos (Zwaan et al., 1995a). Además, estableceremos los aportes de este modelo al estudio de la comprensión de narraciones, así como posibles limitaciones y líneas de desarrollo futuras. 


\section{La comprensión de la narración desde la psicología del discurso: eventos y modelos mentales}

Desde la psicología discursiva, en general, se concibe a la narración como expresiones de experiencias basadas en eventos, construidas o almacenadas cognitivamente y que se organizan en estructuras de conocimiento posibles de ser anticipadas por una audiencia (Graesser, Golding \& Long, 1996). Es decir, se entiende como un recuento producido por mecanismos cognitivos, el cual poseería una estructura en cierta medida convencionalizada. Si bien esta definición es bastante general, sintetiza las teorizaciones que se han producido en las últimas cuatro décadas respecto de esta modalidad.

\subsection{Eventos como base de la narración}

La narración se ha caracterizado por mantener un estatus especial dentro de los estudios en comprensión discursiva, siendo por mucho la que más investigaciones ha generado (Graesser et al., 1996). En efecto, autores que han seguido de cerca el devenir de la corriente del procesamiento del discurso han señalado en más de una oportunidad la excesiva atención que se le ha concedido al estudio de esta (Parodi, 1999; 2005).

Sin embargo, el interés especial del que ha sido objeto no obedece a razones arbitrarias. La abundante investigación al respecto señala que las narraciones serían más fáciles de comprender y recordar que otros modos de organización discursivos (Freedle \& Hale, 1979; Graesser, 1981; Graesser \& Ottari, 1996; Graesser \& Riha, 1984; Rubbin, 1995; Spiro \& Taylor, 1987). Bruner (1986) explica estos resultados planteando que la narración podría corresponder a una 'modalidad del pensamiento', en otras palabras, sería esta una de las formas naturales en que los sujetos organizan sus experiencias y dan sentido a la realidad.

Otra posible explicación corresponde a la planteada por Graesser y colaboradores (1996). Dichos investigadores señalan que una posible razón por la cual la narración se manifiesta como más natural, y por tanto más fácil de comprender, correspondería a que esta describe 'eventos', los cuales a su vez serían experimentados o recreados cotidianamente por los miembros de una comunidad. Desde esta mirada, un 'evento' 
correspondería a una acción que denota un cambio de estado (McNamara \& Magliano, 2009), es decir, acciones dinámicas que se desarrollan en un marco espacio-temporal.

Esta visión, además, postula al 'evento' como unidad central de las representaciones mentales, para lo cual se basa en investigaciones realizadas por Nelson (1986; 1996), Mandler (1984) y Piaget (1952) desde donde se señala que las representaciones mentales más abstractas y los sistemas de razonamiento se derivarían de los conocimientos que poseen los sujetos con relación a dichos 'eventos'. Al respecto, Nelson (1986:4) consigna: "[events] are the initial content of mental representations, (...) stable mental elements (i.e. concepts and categories) are derived from them". Dentro de este marco, cobra aún mayor sentido la afirmación de que la narración sea una modalidad natural del ser humano y que por lo tanto las investigaciones hayan vuelto su mirada preferentemente hacia ella.

\subsection{La noción de modelo mental aplicada a la comprensión de narraciones}

Además del rol fundamental de los 'eventos' dentro de la narración y las representaciones mentales, otro planteamiento que se desprende de la definición de narración expuesta al comienzo de este apartado, dice relación con el hecho de que las narraciones poseerían una estructura posible de predecir por su audiencia. Esta noción se relaciona estrechamente con los conceptos de 'esquemas' (Barttlet, 1932; Kintsch \& Van Dijk, 1978; Johnson \& Mandler, 1980; Mandler \& Johnson, 1977; Rumelhart, 1975, 1977) y 'modelos mentales' (Johnson-Laird, 1983; Van Dijk\& Kintsch, 1983). Dichos conceptos coinciden en que corresponderían, a grandes rasgos, a representaciones estereotipadas construidas en base al conocimiento previo.

La noción de 'esquemas mentales' en la comprensión de la narración se remonta a los estudios respecto a la memoria desarrollados por Bartlett (1932). Este investigador, pionero en los estudios de memoria, postula que los individuos desarrollarían 'esquemas' o patrones mentales de la forma en que se organiza el discurso, en especial de las historias. Dicho autor relaciona esta noción con la memoria reconstructiva y sugiere que podría explicar algunos problemas o distorsiones en la recuperación de información que sufren los individuos, tales como distorsiones, omisiones 
o cambios en la información recuperada. Desde esta teoría, dichos problemas se deberían a que los sujetos utilizan estos 'esquemas' como pistas para la recuperación de lo leído, lo que los llevaría a completar, eliminar o transformar la información de manera que lo reconstruido se ajuste al 'esquema' previamente almacenado.

Siempre dentro de la psicología cognitiva, pero específicamente desde la teoría de la 'gramática de las historias', Mandler y Johnson (1977), entre otros, replantean la concepción de 'esquema' propuesta por Barttlet (1932) por considerarla demasiado general y postulan la existencia de 'esquemas de las historias' (story schemas) los cuales corresponderían a "a set of expectations about the internal structure of stories which serve to facilitate encoding and retrieval" (Mandler \& Johnson, 1977: 112). Como se puede apreciar, las autoras plantean la existencia de unidades dentro de las historias, las cuales se combinarían para formar una estructura canónica, cuya representación mental estereotipada quedaría almacenada en la memoria del individuo y se manifestaría guiando la comprensión y posterior recuerdo de dicha historia.

Algunos de los problemas que presentaron estas concepciones e investigaciones iniciales relativas a los 'esquemas' mentales, y en específico los 'esquemas de las historias' (story schema) dicen relación con la estructura canónica que postulan (Van den Broek \& Gustafson, 1999; Van Dijk, 1999). En efecto, como se aprecia en el análisis planteado en los párrafos anteriores, desde la 'gramática de las historias' se postula que para que una historia sea más comprensible y por ende más fácil de recordar, debe ajustarse al esquema ideal (Mandler \& Johnson, 1977), lo que da como resultado una visión bastante estructurada de la comprensión. Al respecto Van Dijk señala: "such an approach was too neat, too structuralistic and seemed to reflect too little the actually ongoing mental processing. A proper theory of text comprehension and production would have to embody the more dynamic, ad hoc, online, tentative nature of understanding" (1999: 392).

Es dentro de este contexto, entonces, que se planteará la noción más actual de 'modelo de situación', el cual, en una primera aproximación correspondería a "a model the hearer or listener constructs about the situation denoted by the text" (Van Dijk \& Kintsch, 1983: 337). Aquí podemos apreciar un cambio sustancial respecto de la propuesta anterior, esta representación ya no correspondería a una estructura interna del 
texto como lo propone la postura teórica de Mandler y Johnson (1977), sino que corresponde ahora a una representación de la situación descrita por este; en otras palabras, ya no es una representación a nivel estructural la que guía la comprensión, sino una representación de nivel semántico.

Otro cambio importante que introduce esta nueva postura teórica dice relación con el rol central que ocupaba la visión de esquema en la postura anteriormente revisada. Dentro de esta nueva visión, sería el modelo situacional el cual guiaría la comprensión ahora "it is this model which supplies and collects all the relevant information for the adequate comprehension of the text" (Van Dijk \& Kintsch, 1983: 337). El rol del esquema tanto estructural (superestructura) como semántico (macroestructura) pasa ahora a ser visto como un tipo de estrategia utilizada por los lectores para dar sentido a su representación, pero ya no como guía de los procesos de comprensión (Van Dijk \& Kintsch, 1983: 337).

Finalmente, se aprecia que el rol constreñidor y canónico del 'esquema de la historia' desaparece, para dar paso a una visión en la que la comprensión está guiada por representaciones subjetivas de fragmentos de la realidad que el comprendedor posee. Esto implica, además, que la representación mental ya no es contrastada con lo que debería ser el modelo ideal de historia, sino, con lo que el sujeto que comprende se ha representado como el modelo de historia. Desde esta postura, entonces, el modelo resultante sería personal y único, y definiría una interpretación específica de un texto en particular en un momento dado (Van Dijk, 1999).

La noción de 'modelo de situación planteada originalmente por Van Dijk y Kintsch (1983) revisada en los párrafos anteriores, será profundizada y especificada desde varios modelos propuestos en la línea del procesamiento del discurso (Graesser et al., 1994). En el apartado que sigue revisaremos los planteamientos de uno de ellos, el cual ha sido formulado específicamente para dar cuenta de la comprensión de narraciones.

\section{Modelo de indexación de eventos}

De acuerdo a lo mencionado en la introducción, la propuesta de que la comprensión de un texto no sólo implicaría sino que además estaría guiada por la construcción de un 'modelo de situación' (Van Dijk \& Kintsch, 1983) ha sido aceptada ampliamente por los investigadores que trabajan en el área de la psicología discursiva. 
En un intento por llevar este concepto al área específica de la comprensión de narraciones, Zwaan y colaboradores han propuesto el 'modelo de indexación de eventos' (Zwaan et al., 1995a; Zwaan et al., 1995b; Zwaan \& Radvansky, 1998), el cual pretende dar cuenta de la construcción, actualización y recuperación del 'modelo de situación' en la comprensión de narraciones simples (Zwaan et al., 1995b).

Cabe destacar que la centralidad que estos investigadores otorgan al rol del 'modelo de situación' en la comprensión de narraciones nace de la concepción que dichos autores plantean del proceso mismo de comprensión. Para Zwaan y colaboradores llegar a comprender un texto es equivalente a construir una representación situacional coherente del mismo, lo que implicaría que al develar los procesos involucrados en la construcción de dicho nivel de representación los investigadores estarían realmente abordando la pregunta de cómo se comprenden los textos narrativos (Zwaan \& Radvansky, 1998). En otras palabras, este modelo, en apariencia restringido, ha sido concebido con un objetivo final amplio: dar cuenta del proceso de comprensión de narraciones.

Es importante hacer notar que pese a que los investigadores se centran en el 'modelo de situación', no niegan la construcción de otros niveles de representación. De hecho recogen los postulados de Van Dijk y Kintsch (1983) respecto de que serían tres los niveles de representación construidos por los comprendedores. En un primer nivel se encontraría el 'código de superficie' el cual correspondería a una representación verbatim del texto, la cual preservaría su sintaxis y redacción. El segundo nivel correspondería a la 'base textual' la cual es entendida como una red proposicional que contiene los significados extraídos del texto incluyendo las inferencias necesarias para mantener la coherencia local. Finalmente, estaría el 'modelo de situación' como tercer nivel de representación, el cual correspondería a una representación coherente del micro-mundo descrito en el texto (Zwaan et al., 1995a), el cual se define en extenso como: "a mental microworld of events (...), with characters performing actions in the pursuit of goals, events that present obstacles to the goals, conflicts between characters, methods of resolving conflicts, and emotional reactions to the events and conflicts" (Graesser, Louwerse, McNamara, Olney, Cai \& Mitchell, 2007: 290).

Como se aprecia en la definición, este modelo supone que los eventos serían la unidad central sobre la cual se construye el 'modelo de situación' 
(Zwaan, 1999). Dicha asunción no es nueva desde la psicología cognitiva y tiene sus raíces en los postulados revisados en el primer apartado de este trabajo (Nelson, 1986). En dichos postulados se relaciona a un evento con un cambio de estado, lo cual implica una visión dinámica de los mismos, postura que es compartida por Zwaan y colaboradores (Zwaan et al., 1995a; Zwaan et al., 1995b; Zwaan \& Radvansky, 1998). No obstante la centralidad de dicho concepto para este modelo, se aprecia que los autores no proveen una definición precisa de la noción de evento, en cambio, se limitan a señalar una definición operativa, la cual asimila a un evento representacional con un verbo manifestado en el texto (Zwaan, 1999).

En relación a la manera en que estos eventos se representan en la memoria, es decir, respecto de la naturaleza misma de dichos eventos como conjunto de información, los autores postulan que se conformarían de al menos un núcleo (kernel) proposicional y conocimiento episódico pertinente a la situación identificada. Los autores postulan que este núcleo (kernel) proposicional actuaría como la unidad indivisible del evento, no pudiendo ser descompuesta en otras unidades menores (Zwaan, 1999). Es menester señalar que la validez de este postulado plantea dudas, principalmente debido a que virtualmente todo evento puede ser subdivido en eventos menores, hecho que es reconocido por los autores, pero del que señalan no poder dar cuenta en el momento (Zwaan, 1999). En relación al conocimiento episódico que sería parte de estas representaciones, los autores plantean que este estaría determinado en mayor o menor medida por el conocimiento previo del comprendedor, es decir, cuánta experiencia personal o vicaria posean de episodios relacionados y la profundidad en que manejen los mismos, etc. (Zwaan \& Radvansky, 1998). Lo anterior implica que a mayor conocimiento previo, menor será la dependencia del comprendedor respecto del nivel proposicional de la representación, y viceversa, a menor conocimiento previo, el comprendedor se verá obligado a depender en mayor medida del nivel proposicional de la representación (Zwaan, 1999). Cabe señalar que este postulado puede explicar en parte las razones por las cuales una narración aparece como más fácil de comprender que un texto expositivo. En general, los textos expositivos tienen como propósito informar al lector de una situación nueva, por lo que el conocimiento previo que los comprendedores poseen sobre la situación sería menor, a diferencia de una narración (Best, Ozuru, Floyd \& McNamara, 2006), 
la cual, por lo general tiene más similitud con los hechos de la vida cotidiana, lo que permitiría que los comprendedores se enfrenten a ellos con un mayor bagaje de conocimiento, el cual les permitiría construir con mayor facilidad la representación situacional aunque el nivel semántico de la representación no fuese tan completo.

Finalmente queremos señalar que dentro de este modelo, no basta sólo con poseer el conocimiento previo para que este sea utilizado. Los autores postulan que para que este conocimiento sea integrado de manera efectiva en la representación situacional se debe poder activar correctamente (Zwaan \& Radvansky, 1998). Según Zwaan (1999) un factor clave para la activación del conocimiento previo dice relación con las pistas textuales, es decir, con la forma del material lingüístico. Diversas investigaciones han demostrado que factores netamente textuales, como por ejemplo el aspecto del verbo (progresivo o perfectivo) tendría injerencia en la cantidad de conocimiento previo que los sujetos activan respecto de cada evento durante el proceso de comprensión (Magliano \& Schleich, 2000; Radvansky, Zwaan, Federico \& Franklin, 1998; Rinck, Hähnel \& Becker, 2001; Zwaan, 1996).

En resumen, esta postura aborda la comprensión de narraciones desde la construcción de una representación mental del micromundo descrito en el texto, el cual identificaría a los eventos como núcleo central de este modelo situacional. En lo que sigue de este apartado analizaremos en detalle la visión multidimensional que plantea este modelo respecto de dichos eventos, para finalizar señalando algunas limitaciones y desafíos de este planteamiento teórico.

\subsection{Multidimensionalidad del 'modelo de situación'}

Como lo mencionamos en la introducción, desde esta visión se plantea que el 'modelo de situación' sería multidimensional, es decir, estaría compuesto por diferentes categorías de información las cuales el comprendedor monitorea a medida que construye dicha representación (Zwaan et al., 1995a; Zwaan et al., 1995b; Zwaan \& Radvansky, 1998). Estas categorías serían cinco: temporalidad, espacialidad, causalidad, intencionalidad y protagonista (Zwaan et al., 1995b; Zwaan \& Radvansky, 1998).

Queremos señalar que pese a que la elección de estas dimensiones en específico pueda ser motivo de controversia (Therrieault \& Rinck, 2007), 
consideramos que esta concepción multidimensional de construcción de 'modelo de situación' sería uno de los grandes aportes de este modelo. Si bien es cierto es posible rastrear algunas teorizaciones previas que consignaban modelos mentales multicomponenciales (Gernsbacher, 1990; Givón 1993; Nakhimovsky, 1988), las primeras investigaciones empíricas realizadas desde esta mirada fueron desarrolladas por Zwaan y colaboradores (1995a, 1995b) en el marco del modelo de indexación de eventos.

En relación a la elección de estas dimensiones, los autores plantean que estas categorías reflejarían la estructura del mundo real y la manera en que los sujetos interactúan en él, razón por la cual serían las categorías básicas de la comprensión (Zwaan \& Radvansky, 1998). Como evidencia de lo anterior, estos investigadores señalan estudios en los cuales estas dimensiones se han visto reflejadas en la manera en que los sujetos recuerdan eventos autobiográficos, los cuales habrían experimentado directamente (Wagenaar, 1986; Zwaan \& Radvansky, 1998).

Pasamos ahora a revisar las dimensiones básicas propuestas desde este modelo:

1. Temporalidad: La dimensión temporal dice relación con la información respecto del marco temporal en el que se desenvuelve la narración que se extrae del texto (Zwaan et al., 1995a; Zwaan et al., 1995b; Zwaan \& Radvansky, 1998). Según estos autores, los sujetos no podrán comprender a cabalidad la situación descrita en un texto a menos que puedan extraer la información relativa al tiempo en que estos eventos ocurren (Zwaan \& Radvansky, 1998). Lo anterior cobra especial relevancia dentro de la comprensión de los eventos constitutivos de una narración puesto que, como ya se ha señalado en el transcurso de este trabajo, dichos eventos son entendidos desde esta perspectiva como compuestos de un cambio de estado, el cual necesariamente se debe desarrollar en un marco temporal (Magliano \& Schleich, 2000).

2. Espacialidad: La dimensión espacial dice relación con la información respecto del escenario o lugar en el cual se desarrollan los eventos que constituyen la narración (Zwaan et al., 1995a; Zwaan et al., 1995b; Zwaan \& Radvansky, 1998). Los autores incluyen esta dimensión basándose en la extensa bibliografía que señalaría que los comprendedores monitorean el esquema espacial en el que se mueven 
los protagonistas de las narraciones mientras leen un texto narrativo (Glenberg, Meyer, \& Lindem, 1987; Morrow, Bower, \& Greenspan, 1989; Morrow, Greenspan, \& Bower, 1987; Rinck \& Bower, 1995; Wilson, Rinck, McNamara, Bower, \& Morrow, 1993), sin embargo, los autores advierten que dichas investigaciones no proveen evidencia de que esta dimensión se monitoree de forma espontánea, debido a que las investigaciones señaladas requerían en primer lugar que los sujetos memorizaran un mapa del lugar que posteriormente serviría de escenario para la narración (Zwaan et al., 1995a; Zwaan et al., 1995b; Zwaan \& Radvansky, 1998). Es importante destacar que la inclusión de esta dimensión como una de las dimensiones básicas para la construcción, actualización y posterior recuperación del modelo de situación, ha sido bastante discutida, principalmente debido a que se ha encontrado evidencia empírica que apunta a que cuando los lectores no poseen el beneficio de un mapa, no monitorearían dicha dimensión (Hakala, 1999; Langston, Kramer \& Glenberg, 1998; Zwaan \& Van Oostendorp, 1993)

3. Causalidad: Esta dimensión dice relación con las representaciones de las relaciones causales que existen entre los eventos descritos en el texto. Es importante señalar en este punto que dichas relaciones no necesariamente deben estar explicitadas en el texto, es decir, no sería necesaria la existencia de conectores para señalar la misma, sin embargo, se ha encontrado evidencia de que un mayor número de conectores contribuye a una mayor coherencia en la representación final de la situación. Algunos investigadores han planteado que esta sería la dimensión principal sobre la cual se construye el modelo de situación (Trabasso \& Sperry, 1985; Trabasso \& Suh, 1993), sin embargo, Zwaan y colaboradores postulan a esta categoría informacional como solo una más de las dimensiones básicas a ser consideradas por los sujetos (Zwaan et al., 1995a; Zwaan et al., 1995b; Zwaan \& Radvansky, 1998).

4. Intencionalidad: De acuerdo a los autores, esta dimensión dice relación con las metas que el protagonista de una narración trata de alcanzar y por lo tanto con las motivaciones que sustentan el desarrollo de los eventos (Zwaan et al., 1995a; Zwaan et al., 1995b; Zwaan \& Radvansky, 1998). Los autores afirman que esto correspondería a un comportamiento natural ya que "human behavior is goal directed, and because narratives describe human behavior, readers can use general cognitive procedures to explain human behavior" (Zwaan \& 
Radvansky, 1998: 173). Estos investigadores apoyan su decisión de incluir esta categoría de información en estudios que afirman que los sujetos mantendrían en mente los propósitos que mueven a los protagonistas hasta que estos sean alcanzados (Suh \& Trabasso, 1993; Trabasso \& Suh, 1993; Lutz \& Radvansky, 1997).

5. Protagonista: Esta categoría dice relación con los argumentos, ya sea protagonistas u objetos, sobre los cuales giran los eventos descritos en la narración (Therriault \& Rinck, 2007). Evidencia para incluir esta categoría informacional proviene de diversas investigaciones que señalan que los lectores monitorearían la identidad y los rasgos de los protagonistas durante la comprensión (Albrecht \& O'Brien, 1993, 1995; Cook, Halleran \& O’Brien, 1998; O’Brien, Rizzella, Albrecht \& Halleran, 1998) y de las investigaciones realizadas en resolución de anáforas (Garrod \& Sanford, 1977; Sanford, Moar \& Garrod, 1988).

Según los autores de este modelo, existen dos hipótesis o supuestos principales que subyacen a los planteamientos antes señalados. Estas son de especial interés para el presente trabajo investigativo, ya que repercuten directamente en los postulados que desde esta visión se plantean respecto de la facilidad de la comprensión y el posterior recuerdo de textos narrativos, razón por la cual dedicaremos unas líneas a su revisión.

Hipótesis 1: Igualdad de injerencia de las dimensiones: En primer lugar se asume que las dimensiones nombradas tendrían igualdad de peso o injerencia respecto de la construcción del 'modelo de situación', es decir, no habría una dimensión central que guíe el proceso antes señalado. Cabe señalar que los investigadores modificaron dicha aseveración en sus últimos artículos (Zwaan \& Radvansky, 1998; Zwaan, 1999) y proponen que la injerencia de cada dimensión sería regulada por los sujetos comprendedores respecto de las metas que persiguen, vale decir, si su objetivo de comprensión dice relación con la necesidad de formar una representación del esquema espacial, es muy probable que sea la dimensión espacial en torno a la que giren la construcción de la representación situacional. No obstante, se hace necesario señalar que el grueso de la investigación realizada por Zwaan y colaboradores (1995a, 1995b), y que sería el principal sustento del modelo de indexación de eventos, fue concebida y llevada a cabo bajo el supuesto de igualdad antes mencionado. 
Es importante recordar que para este modelo el resultado de la comprensión será una representación coherente almacenada en la memoria. Dicha representación correspondería a "a network of nodes that code the events described in and inferred from the story" (Zwaan \& Radvansky, 1998:179), en otras palabras, el 'modelo de situación'. Para estos investigadores, dos nodos correspondientes a eventos distintos se podrían conectar a través de un número dado de enlaces relacionales, o continuidad dimensional. A modo de ejemplo podemos señalar que si dos nodos comparten una dimensión de protagonista (agente) o de tiempo, se entiende que estos eventos serían continuos respecto de dichas dimensiones (temporal o de protagonista). En este sentido, los investigadores plantean que la fuerza de las interconexiones entre los nodos que representan los eventos de la historia en la memoria será una función del número de dimensiones que compartan dichos eventos, es decir, mientras más relacionados estén los eventos respecto de estas dimensiones, mayor será el recuerdo de los sujetos de esa historia (Zwaan, 1999; Zwaan et al., 1995a; Zwaan et al., 1995b; Zwaan \& Radvansky, 1998).

La evidencia empírica que sustenta dicha hipótesis proviene de diferentes investigaciones realizadas por Zwaan y colaboradores durante la década del noventa, las cuales usando el método de 'agrupamiento de verbos' (verb clustering) y 'tareas de reconocimientoprimed' (primed-recognition tasks) encontraron una correlación positiva entre los eventos con mayor continuidad situacional, es decir los que se solapan en un mayor número de dimensiones, y la fuerza de la representación de dichos eventos en el 'modelo situacional' construido por dichos sujetos (Radvansky, Zwaan, Federico \& Franklin, 1998; Radvansky \& Zacks, 1991; Zwaan, 1996; Zwaan et al., 1995a, Zwaan et al., 1998).

Hipótesis 2: Dicotomía de las dimensiones: Una segunda hipótesis que subyace este modelo dice relación con que las dimensiones que componen cada evento se constituirían en categorías dicotómicas lo que implica que "either two events are related on a dimension or they are not" (Zwaan, 1999:98). Siguiendo esta lógica no sería importante, por ejemplo, si dos eventos descritos en un texto se encuentran a minutos o años de distancia ya que el solo hecho de que exista un cambio en la dimensión temporal sugiere un quiebre de continuidad y, por lo tanto, los comprendedores deberían mostrar el mismo 
comportamiento al enfrentarse a ambas situaciones recién descritas. Cabe señalar que al igual que el supuesto anterior, esta visión dicotómica ha generado bastante controversia lo que ha llevado a los autores a plantear que esta supuesta dicotomía se trataría de una decisión metodológica, pero que se hace necesaria mayor cantidad de investigación para poder llegar a una determinación respecto de esta problemática (Zwaan, 1999).

El supuesto recién planteado cobra especial relevancia si se considera que desde este modelo se predice que la continuidad o discontinuidad que muestra cada evento respecto del anterior en relación a cada dimensión, sería un indicador tanto de la fuerza con que este evento será anclado en la memoria a largo plazo como de la facilidad con que este evento será procesado.

Esta hipótesis se relaciona directamente con la actualización del 'modelo de situación' y por consiguiente con su facilidad de procesamiento. De acuerdo a los autores, el proceso de actualización del modelo de situación (es decir, la integración de la información entrante al modelo situacional construido en ese momento) se verá dificultado si la información entrante presenta discontinuidad en una o más dimensiones respecto del modelo en construcción. En este sentido, mientras más dimensiones deban ser actualizadas durante la comprensión de un evento, mayor será el tiempo que tomará su procesamiento, es decir, mientras más continua sea la narración respecto de su marco espaciotemporal, causalidad, protagonistas y las intenciones de ellos, será más fácil de comprender (Zwaan \& Radvansky, 1998; Rinck \& Weber, 2003).

Evidencia de apoyo a dicha hipótesis proviene mayormente de estudios que se han realizado controlando sólo la injerencia de una dimensión sobre los procesos comprensivos (Graesser, 1981; Morrow et al., 1987; Suh \& Trabasso, 1993; Zwaan, 1994, 1996; Zwaan, Madden \& Whitten, 2000), consecuentemente, las investigaciones realizadas por Zwaan y colaboradores (1995a, 1995b) se constituyen como pioneras en la indagación de la injerencia de la discontinuidad en múltiples dimensiones. Dichos estudios establecieron que al menos la dimensión causal y temporal tienen efectos aditivos sobre el tiempo de lectura, es decir si un evento es discontinuo en estas dos dimensiones su procesamiento requerirá de mayor esfuerzo que si fuese discontinuo en solo una dimensión (Zwaan \& Radvansky, 1998). 
Adicionalmente, los investigadores concluyeron que cada dimensión "madea unique contribution toward explaining variancein the relatedness scores for story events" (Zwaan \& Radvansky, 1998:179), es decir, pese a que los efectos de la discontinuidad situacional sería sumativo, estos autores afirman que las dimensiones no estarían conectadas entre ellas.

En resumen, podemos señalar que este modelo plantea una visión de la comprensión de narraciones basada en el supuesto de que el sistema cognitivo presentaría una propensión natural hacia la percepción de eventos dinámicos. Lo anterior explicaría la naturalidad y facilidad en la comprensión de la narración, la cual es entendida justamente como compuesta de estos eventos. En consecuencia, los investigadores le otorgan un papel especial a los verbos descritos en el texto los cuales actuarían como pistas textuales que delimitarían dichos eventos y denotarían su dinamicidad. Otro aspecto a destacar dice relación con que desde esta visión se supone que la comprensión de una narración implica la construcción de una representación de la situación descrita en el texto cuya coherencia se establecería a través de la continuidad de cinco dimensiones situacionales. Esta continuidad, además, determinaría la fuerza del recuerdo de dicha representación, y la facilidad de su procesamiento.

\subsection{Limitaciones del modelo de indexación de eventos}

En primer lugar queremos señalar que una de las principales limitaciones de este modelo dice relación con que asume que las dimensiones propuestas serían independientes entre sí, en otras palabras, los efectos de una dimensión no afectarían a las demás. Contrario a este supuesto, se ha encontrado evidencia empírica que demostraría que el efecto de una dimensión incide en otras. Dentro de estos estudios podemos señalar a Rinck y Bower $(2000,2004)$ quienes a través de una serie de experimentos encontraron que la accesibilidad de la dimensión espacial se vería afectada por variables de la dimensión temporal. Otras interrelaciones han sido reportadas en diferentes estudios, por ejemplo interrelación protagonista-temporalidad-espacialidad (Magliano, Miller \& Zwaan, 2001; Rinck y Weber, 2003).

Otra limitación respecto de este modelo dice relación con el supuesto planteado por Zwaan y colaboradores (1995a, 1995b, 1998), el cual asume que todas las dimensiones tendrían una injerencia igualitaria respecto 
de la construcción del 'modelo de situación' de una historia. Se ha encontrado evidencia de que el efecto de la discontinuidad dimensional sobre el tiempo de lectura de las historias no sería homogéneo, lo que lleva a estos investigadores a concluir que habría dimensiones que tienen dominancia sobre otras, en especial las dimensiones temporal y de protagonista (Rinck \& Hähnel, 2002; Rinck \& Weber, 2003; Zwaan, Radvansky, Hilliard \& Curiel, 1998).

En línea con lo anterior, algunos autores han postulado que las dimensiones espacial e intencional serían 'second-order dimensions' (Therriault \& Rinck, 2007), es decir, no tendrían la predominancia de las demás categorías de información, debido a que se ha encontrado que su monitoreo depende de otros factores como la tarea o meta que el comprendedor esté tratando de cumplir.

Finalmente queremos señalar que este modelo ha sido solo probado en la comprensión de narraciones simples y con historias en su mayoría creadas o editadas por los investigadores, razón por la cual no se puede considerar aún como una teoría general de la comprensión de narraciones. No obstante, se constituye en una valiosa herramienta teórica y metodológica desde donde cimentar estudios posteriores. Como evidencia de lo anterior podemos señalar diversos estudios que han investigado la comprensión de narraciones a partir de las dimensiones señaladas por Zwaan y colaboradores, aunque sin adoptar completamente este modelo (Rinck \& Hähnel, 2002; Rinck \& Weber, 2003), además, queremos destacar que este modelo se ha extrapolado con el fin de ser usado en estudios relacionados con la comprensión de secuencias de eventos en formato audiovisual (ej. secuencias cinematográficas) (Magliano et al., 2001; Zacks, Speer, \& Reynolds, 2009).

\section{Reflexiones finales}

Desde un enfoque cognitivo la narración es vista como natural al ser humano ya que se presenta como una manera que poseen los sujetos para dar sentido a la experiencia, segmentándola y categorizándola. Revisamos varias de estas posturas, desde Bruner (1986), Mandler y Johnson (1977) y finalmente la posición de la psicología discursiva (Zwaan et al., 1995a; 1995b) que nos propone la segmentación natural de la realidad a partir de eventos, los cuales a su vez estarían constituidos por estas dimensiones de información ya mencionados y analizados. 
Un aporte del modelo de indexación de eventos dice relación con que presenta una base teórica y empírica que ha permitido a otros investigadores continuar el avance en la investigación de la comprensión de narraciones desde la perspectiva del monitoreo e integración de las diversas dimensiones. Se aprecia que su mayor aporte se encuentra en plantear y comprobar empíricamente esta visión de multimodalidad en las representaciones, ya que, como se mencionó a lo largo de la revisión, la arquitectura cognitiva planteada y la naturaleza de las representaciones que postula no poseen suficiente solidez empírica o sustento teórico profundo.

Este modelo presenta desafíos metodológicos importantes para la investigación en la comprensión de narraciones ya que, a la fecha, se aprecia una falta de metodologías experimentales que permitan aislar las diferentes dimensiones postuladas para así llegar a conclusiones decidoras respecto de la injerencia que estas tendrían en los procesos comprensivos de los sujetos.

Finalmente, cabe señalar la gran cantidad de evidencia empírica que sustenta varios de los postulados de este modelo de comprensión de narraciones. Sin duda, este hecho hace necesario que quienes trabajan en el área de la didáctica de la comprensión textual tengan conocimiento de sus postulados, de manera de considerarlos a la hora de planificar e implementar intervenciones o planes de mejoramiento del desarrollo de la comprensión textual. 


\section{Referencias bibliográficas}

Albrecht, J. \& O'Brien, E. (1993). Updating a mental model: Maintaining both local and global coherence. Journal of Experimental Psychology: Learning, Memory and Cognition 19. 1061-1070.

Albrecht, J. \& O'Brien, E. (1993). Goal processing and the maintenance of global coherence. En R. Lorch \& E. O'Brien (Eds.) Sources of coherence in reading. Hillsdale: Erlbaum. 263-278.

Bransford, J., Barclay, J. \& Franks, J. (1972). Sentence memory: A constructive versus interpretive approach. Cognitive Psychology 3. 193-209.

Bransford, J. \& Johnson, M. (1972). Contextual pre-requisites for understanding: some investigations of comprehension and recall. Journal of Verbal Learning and Verbal Behavior 11. 717-726.

Best, R.; Ozuru, Y.; Floyd, R. \& McNamara, D. (2006). Children's text comprehension. Effects of genre, knowledge, and text cohesion. En S. Barab, K. Hay, D. Hickey (Eds.) Proceedings of the Seventh International Conference of the Learning Sciences. Mahwah: Erlbaum. 37-42.

Cook, A.; Halleran, J. \& O'Brien, E. (1998). What is readily available during reading? A memory based view of text processing. Discourse Processes 23. 169-192.

Freedle, R. \& Hale, G. (1979). Acquisition of new comprehension schemata for expository prose by transfer of a narrative schema. En R. Freedle (Ed.). New directions in discourse processing. Norwood: Ablex.

Garrod, S. \& Sanford, A. (1977). Interpreting anaphoric relations: The integration of semantic information while reading. Journal of Verbal Learning and Verbal Behavior 16. 7790. 
Gernsbacher, M. (1990). Language comprehension as structure building. Hillsdale: Erlbaum.

Givón, T. (1993). Coherence in text, coherence in mind. Pragmatics and Cognition 1. 171-227.

Graesser, A. \& Ottati, V. (1996). Why stories? Some evidence, questions, and challenges. En R. Wyer (Ed.). Knowledge and memory: The real story. Hillsdale: Erlbaum. 121-132

Graesser, A. \& Riha, J. (1984). An application of multiple regression techniques to sentence reading times. En D. Kieras \& M. Just (Eds.). New methods in comprehension research. Hillsdale: Erlbaum.

Graesser, A., Singer, M. \& Trabasso, T. (1994). Constructing inferences during narrative text comprehension. Psychological review 101 (3). 371-395.

Graesser, A.; \& Wiemer-Hastings, K. (1999). Situation models and concepts in story comprehension. En S. Goldman, A. Graesser \& P. van den Broek (Eds). Narrative comprehension, causality, and coherence. Mahwah: Erlbaum. 77-92

Hakala, C. (1999). Accessibility of spatial information in a situation model. Discourse Processes 27 (3). 261-279.

Johnson-Laird, P. (1983). Mental models. Cambridge: Cambridge University Press.

Johnson, N. \& Mandler, J. (1980). A tale of two structures: underlying and surface form in stories. Poetics 9. 51-86.

Magliano, J.; Miller, J. \& Zwaan, R. (2001). Indexing space and time in film understanding. Applied Cognitive Psychology 15. 533-545.

Magliano, J. \& Schleich, M. (2000). Verb aspect and situation models. Discourse Processes 29 (2). 83-112.

Mandler, J. (1978). A code in the node: The use of a story schema in retrieval. Discourse Processes 1. 14-35. 
Mandler, J. \& Goodman, M. (1982). On the psychological validity of story structure. Journal of Verbal Learning and Verbal Behavior 21. 507-523.

Mandler, J. \& Johnson, N. (1977). Remembrance of things parsed: Story structure and recall. Cognitive Psychology 9. 111-151.

McKoon, G. \& Ratcliff, R. (1992). Inference during reading. Psychological Review 99 (3). 440-466.

Morrow, D.; Bower, G. \& Greenspan, S. (1989). Updating situation models during narrative comprehension. Journal of Memory and Language 28. 292-312.

Morrow, D.; Greenspan, S. \& Bower, G. (1987). Accessibility and situation models in narrative comprehension. Journal of Memory and Language 26. 165-187.

Nakhimovsky, A. (1988). Aspect, aspectual class, and the temporal structure of narrative. Computational Linguistics 14 (2). 29-43.

Nelson, K. (1986). Event knowledge: Structure and function in development. Hillsdale: Erlbaum.

O'Brien, E.; Rizzella, M.; Albrecht, J. \& Halleran, J. (1998). "Updating situational models," Journal of Experimental Psychology: Learning, Memory and Cognition 24, 1200-1210.

Radvansky, G. \& Zacks, R. (1991). Mental models and the fan effect. Journal of Experimental Psychology: Learning, Memory and Cognition 17. 940-953.

Radvansky, G.; Zwaan, R.; Federico, T. \& Franklin, N. (1998). Retrieval from temporally organized situation models. Journal of Experimental Psychology: Learning, Memory and Cognition 24, 1224-1237.

Rinck, M.; Hähnel, A. \& Becker, G. (2001). Using temporal information to construct, update and retrieve situation models of narratives. Journal of Experimental Psychology: Learning, Memory and Cognition 27. 67-80. 
Rinck, M. \& Weber, U. (2003). Who when where: An experimental test of the event-indexing model. Memory and Cognition 31 (8). 1284-1293.

Rubin, D. (1995). Memory in oral traditions: The cognitive psychology of epic, ballads, and counting-out-rhymes. New York: Oxford University Press.

Rumelhart, D. (1975). Notes on a schema for stories. En D. Robrow \& A. Collins (Eds.) Representation and understanding: studies in cognitive science. New York: Academic Press.

Sanford, A. \& Garrod, S. (1981). Understanding written language. Chichester: John Wiley \& Sons.

Stein, N. \& Glenn, C. (1979). An analysis of story comprehension in elementary school children. En R. Freedle (Ed.). New directions in discourse processing. Norwood: Ablex.

Thorndyke, P. (1977). Cognitive structures in comprehension and memory of narrative discourse. Cognitive Psychology 9. 77110.

Van Dijk, T. \& Kintsh, W. (1983). Strategies of discourse comprehension. New York: Academic Press.

Wagenaar, W. (1986). My memory: A study of autobiographical memory over six years. Cognitive Psychology 18 (2). 225-252.

Yarkoni, T.; Speer, N.; Zacks, J. (2008). Neural substrates of narrative comprehension and memory. NeuroImage 41 (4). 1408-1425.

Zacks, J.; Speer, N. \& Reynolds, J. (2009). Segmentation in reading and film comprehension. Journal of Experimental Psychology: General 138 (2). 307-327.

Zwaan, R. (1996). Processing narrative time shifts. Journal of Experimental Psychology: Learning, memory, and cognition 22. 1196-1207. 
Zwaan, R. (1999). Five dimensions of narrative comprehension: The event-indexing model. En S. Goldman, A. Graesser \& P. Van den Broek (Eds). Narrative comprehension, causality, and coherence. Mahwah: Erlbaum. 93-110

Zwaan, R.; Langston, M. \& Graesser, A. (1995a). The construction of situation models in narrative comprehension: an event-indexing model. Psychological science 6 (5). 292-297.

Zwaan, R.; Madden, C. \& Whitten, S. (2000). The presence of an event in the narrated situation affects its availability to the comprehender. Memory E Cognition 28, 1022-1028.

Zwaan, R.; Magliano, J. \& Graesser, A. (1995b). Dimensions of situation model construction in narrative comprehension. Journal of Experimental Psychology: Learning, Memory, and Cognition 21, 386-397.

Zwaan, R. \& Radvansky, G. (1998). Situation models in language comprehension and memory. Psychological Bulletin 123 (2). 162-185.

Zwaan, R.; Radvansky, G.; Hilliard, A. \& Curiel, J. (1998). Constructing multidimensional situation models during reading. Scientific Studies of Reading 2, 199-220.

Zwaan, R. \& Van Oostendorp, H. (1993). Do readers construct spatial representations in naturalistic story comprehension? Discourse processes 16, 125-143.

Zwaan, R.; Radvansky, G. \& Whitten, S. (2002). Themes and situation models. En M. Louwerse \& W. van Peer (Eds.). Thematics: Interdisciplinary studies. Amsterdam: John Benjamins. 35-53.

Zwaan, R.; Stanfield, R. A. \& Yaxley, R. H. (2002). Language comprehenders mentally represent the shapes of objects. Psychological Science 13 (2). 168-171. 